\title{
Quantitative Analysis of Breast Shapes
}

\author{
Rong Zheng ${ }^{\star a, b}$, Winnie Yu ${ }^{a}$, Jintu Fan ${ }^{a}$ \\ ${ }^{a}$ Institute of Textiles and Clothing, Hong Kong Polytechnic University, Kowloon, Hong Kong; \\ ${ }^{\mathrm{b}}$ Aimer Human Engineering Research Centre, Beijing Institute of Fashion Technology, Beijing, China
}

\begin{abstract}
Breast shape analysis underpins the development of bra sizing and bra cup pattern making. Our previous study established a new bra sizing system using two key measurements -- underbust girth and breast depth width ratio (DWR), based on the analysis of 103 3D and manual breast measurements of 456 subjects. To set up a useful link between the new breast sizing and bra pattern development, this study is devoted to 1) investigate the breast shapes quantitatively according to the new bra sizing, 2) explore the key measurements that can be used for bra pattern cutting, 3) search additional measurements that can help the new bra sizing system to be used more efficiently. The correlation coefficient analysis was conducted on the 103 measurements among the most-frequently used 14 bra sizes in the market. 40 measurements having higher correlation coefficients more than 0.5 with the DWR were identified to further improve the application effect of the new bra sizing system in the practical bra design and manufacture. By means of factor analysis, five factors describing the breast shape were derived based on the 40 measurements. Eight measurements closely related to the bra pattern making were obtained. Moreover, the width of the breast and the depth of the breast were added into the new bra sizing system as the control measurements. The effective reference means of eight measurements were obtained for the bra pattern making with the explore statistic analysis.
\end{abstract}

Keywords: Breast shapes, 3D body scan data, breast sizing, quantitative analysis, breast measurements, bra pattern development

\section{Introduction}

A good bra plays an essential role for women to achieve an aesthetically pleasing shape, while providing the necessary support and ensuring comfort. However, some researches [1, 2, 3] revealed that $70 \%$ British women wore the wrong-sized bra, especially women with large breasts. Greenbaum et al. [4] carried out a study on 102 women, and found that most of the bra sizes that manufacturers recommended for their measurements did not match with their breast shapes. Most of the subjects wore the bra with undersized band and oversized cup. The more obese they were, the more remarkable the disparity between the recommended and actual band size was.

Breast shape measurements and classifications are the foundation of bra sizes and bra cup pattern. Studies about body shape have been made to establish clothing design standards [5]. In contrast, few researches have been conducted to examine the breast shape for the bra design, though numerous efforts have been made to measure breast volume for asymmetry analysis and breast surgery purpose $[6,7,8,9]$.

In light of the side- view of breast curve, Martin categorized the breast shapes into four types: flat breast (breast height $=3$ to $5 \mathrm{~cm}$ ), hemisphere breast (breast height $=5 \mathrm{~cm}$ to $6 \mathrm{~cm}$ ), conic breast (breast height $>6 \mathrm{~cm}$ ), and goat drooping breast [10].

To integrate beauty and comfort into the intimate apparel, Wacoal [11] found 6 key parameters of female's body beauty, based on manual measurements and 3-D scan data of 1115 Japanese women. They include the proportion of BWH (bust, waist and hip) circumference, width and thickness of torso, base shape and orientation of the breast, base shape and orientation of the hip, balance between whole height \& weight, proportion balance of height. Wacoal also defined 7 female body types considering the front view and side view images [12].

Medical researchers have made lots of efforts to explore new ways for breast volume measurements. In an attempt to evaluate accuracy of different methods for breast volume measuring, Bulstrode et al. [13] compared five techniques to assess breast volumes of 10 women. Volume measurements obtained from mammograms, thermoplastic moulding, magnetic resonance imaging, Archimedes principle and anatomical measurements, were compared. They concluded that the breast volume from mammography was reasonably accurate, and thermoplastic moulding showed promise and should be further studied as it gives not only a volume assessment but a three-dimensional impression of the breast shape that would be valuable in assessing effect of breast-conserving-surgery. 
Given that there is no widely-recognized method for breast volume calculation, Kovacsa et al [14] compared breast volume calculations with 3D scanning and three classic methods, namely nuclear magnetic resonance imaging (MRI), thermoplastic castings and anthropomorphic measurements. The results showed that MRI presented the highest measurement precision, with a mean deviation of $1.5670 .52 \%$, compared with $2.2770 .99 \%$ for the $3 \mathrm{D}$ scanner, $7.9773 .53 \%$ for thermoplastic castings, and $6.2671 .56 \%$ for the anthropomorphic measurements. Breast volume calculations using MRI showed the best agreement with 3D scanning measurement, followed by anthropomorphic measurement and thermoplastic castings. Then the conclusion was drawn that $3 \mathrm{D}$ scanning provided an acceptable accuracy for breast volume measurements.

In the apparel industry, Lee et al. [15], by means of 3D scanning technique, developed a folding line method to determine a continuous and natural boundary for the breasts so that the more precise measurements of the breast base and volume can be achieved.

In an effort to design the ergonomic brassiere wire fitting to the curve of under breast and thorax and apply the 3D measurement of human body structure to the product design and evaluation by means of phase shifting moiré topography, Lee \& Hong [16] sampled 21 middle-aged women in the subjective wear trial, and found that the fitting of the global average radius of wire curvature to the bodyline of the under breast curve was an important variable for the bra underwire design. While conventional shape of brassiere wire was suitable to the majority of the subjects, for those with skewed under breast lines, the wire with larger global average radius of curvature along the inner part of the under breast line was rated higher in terms of comfort and appearance. The authors recommended a $0.015 \mathrm{~N} / \mathrm{mm}^{2} \mathrm{~mm}$ magnitude of the torsional rigidity of the brassiere wire.

In spite of all the achievements on breast shape analysis and breast volume studies, the existing problem is still striking: no practical correlation between the bra design and breast shapes has been established. In previous researches, several important aspects of the bra design process have been identified, including fabric selection, pattern development and grading, of which the last two procedures are relevant to the breast shape. So it is quite necessary to investigate the breast shapes and to build up a relationship between the breast sizing and bra patterns.

On the basis of our previous studies on exploring a new method to describe the nature breast boundary depending on the vertical sections of the breast, and developing a new bra sizing system [17, 18], which were both based on the analysis of the $1033 \mathrm{D}$ and manual nude breast measurements of 456 subjects aged between 20 and 39, this study attempts to establish a practical link between the new breast sizing and bra pattern development, with the specific purposes as follows:

1) to investigate the breast shapes quantitatively according to the new bra sizing;

2) to explore the key measurements that can be used for bra pattern cutting;

3) to find additional measurement that can help the new bra sizing system to be used more efficiently.

\section{Review of a new bra sizing system}

The current Chinese bra sizing system (FZ/T 73012-2004) is a metric sizing system, similar to that used in US, Europe and Japan, structured with underbust girth and the difference between bust girth and underbust girth. In this system, the bra size number directly manifests the underbust girth, and the cup volume is indicated by the centimeter difference between the full bust and the underbust measurements. For example, the bra size $75 \mathrm{~A}$ indicates the underbust girth of $75 \mathrm{~cm}$ and a $10 \mathrm{~cm}$ difference between bust girth and underbust girth. From AA cup to $G$ cup, the differences from $7.5 \mathrm{~cm}$ to $25.0 \mathrm{~cm}$ are indicated respectively, with a $2.5 \mathrm{~cm}$ interval between each other.

For the shortcomings of the existing bra sizing system, like lack of scientific basis, lack of accuracy and failure to distinguish various breast shapes within the same size group [19], a new bra sizing was proposed by Zheng et al. [18].

Principal components factor analysis was conducted to determine the key parameters for the description of breast shapes. The analysis results revealed that eight factors seemed to be the most ideal, which could explain about $80 \%$ of the total sample variance, with the first two factors, overall body build and volume of the breast, accounting for $43.26 \%$ of the total variance. Therefore, to achieve a clear structure of the new sizing system, the two most important key variables in forming factor 1 and factor 2, namely underbust girth and breast depth width ratio (DWR), were identified to classify the breast shapes in the new bra sizing system.

The $5 \mathrm{~cm}$ size interval of the underbust girth in the existing bra sizing system remains in the new bra sizing system. A layered clustering method based on underbust groups was conducted to categorize breast shapes. In the study, 465 subjects were first divided into eight underband categories from $65 \mathrm{~cm}$ to $100 \mathrm{~cm}$ with a $5 \mathrm{~cm}$ interval. A hierarchical clustering was first made, and then a layered K-means cluster analysis was conducted using the variable DWR, referring to the preliminary hierarchical results. 
Finally, the eight new bra sizes describing the breast shapes were obtained in light of the value of DWR for each underband category, namely $0.25,0.30,0.34,0.38,0.42,0.46,0.50$ and 0.55 , respectively corresponding to the cups, $A A^{*}, A^{*}, B^{*}, C^{*}, D^{*}, E^{*}, F^{*}, G^{*}$.

According to the anthropometric data of 456 subjects, about $14(3 \%)$ subjects were not covered in the existing bra sizing system; in contrast, only five subjects (1\%) were not covered in the new bra sizing system. The analysis results indicated that the two-dimensional probability coverage rate of the new system was $97.43 \%$ (see figure 2.), better than $94.29 \%$ (see figure 1.) achieved by the existing system. If the bra categories of $65 \mathrm{~cm}$ underbands, $A A\left(A A^{*}\right)$ cups and $G\left(G^{*}\right)$ cups were excluded, which are generally not included in manufacturing, the partial probability coverage rate of the new system showed a much higher percentage (84.86\%), much superior than $77.19 \%$ of the existing sizing system. Besides, in the assessment in terms of efficiency of classifying breast shapes and fit performance for the covered individuals, the new sizing system also showed superiority over the existing system, while maintaining a clear structure and keeping the same number of size categories [20].

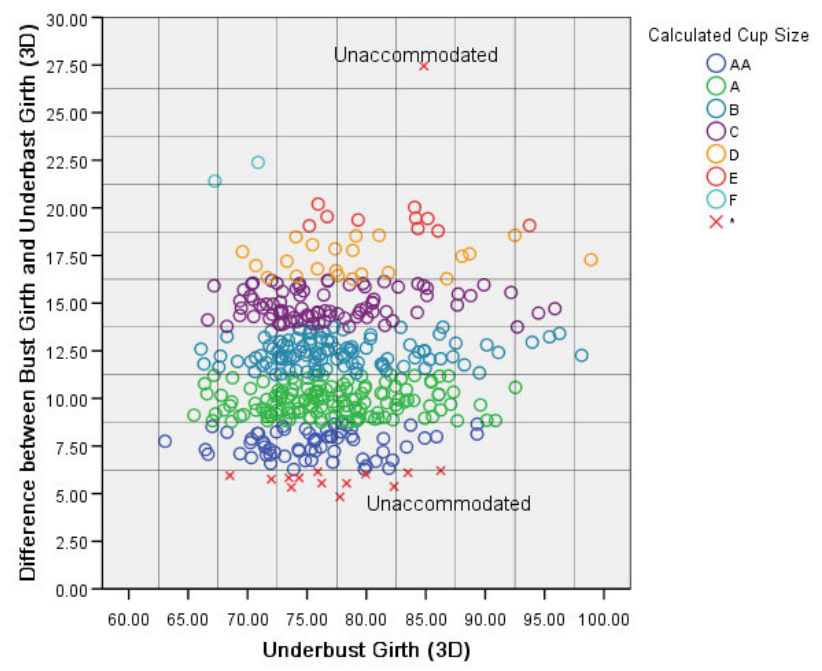

Fig. 1. The current bra size categories based on 456 subjects

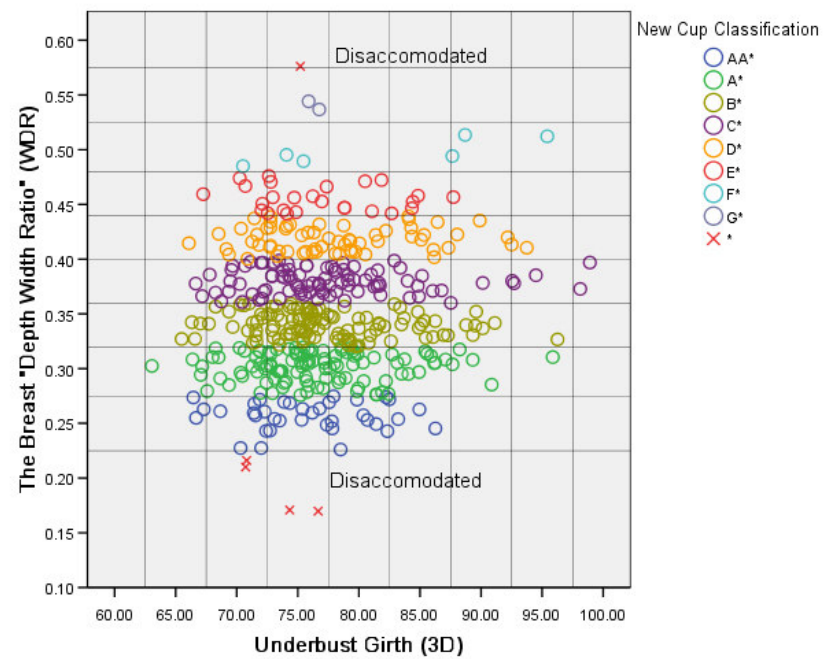

Fig. 2. The new bra size categories for 455 subjects

For all the merits of the new sizing system, there still exists some room for improvement. For example, in the size $75 \mathrm{~B}^{*}$, the value of DWR is 0.34 , but there can be different cases for it as DWR is a ratio of the breast depth and the breast width, and its value can be the same with the depth and width increased or decreased at the same rate. Are there any new approaches that can solve this problem? Are there any key measurements that can be used as key parameters to link the breast shapes and the bra pattern construction together? This paper tries to identify the additional measurements that can help the new bra sizing system to be used more efficiently. 


\section{Methodology}

\subsection{Selection of subjects}

For the bra manufacturers, not all the sizes in a bra sizing system will be their concern. Usually they only produce the bras with the sizes of high population coverage, and the bra paper pattern design is also targeted at those highly-covered sizes. To discuss the relationship between the breast shape and bra paper pattern design more conveniently, based on the new bra sizing system, the study selected several highly-covered sizes, meanwhile considering those bra sizes which occupy the relatively high market shares in the practical manufacturing and sale [18]. The sizes involved in this study include $70 A^{*}-70 C^{*}, 75 A^{*}-75 D^{*}, 80 A^{*}-80 D^{*}, 85 B^{*}-D^{*}$. 322 subjects were selected out of the data set containing 456 subjects. The sizes, $75 \mathrm{~A}^{*}, 75 \mathrm{~B}^{*}$ and $75 \mathrm{C}^{*}$, are the top three as far as the population coverage is concerned, respectively accounting for $13.4 \%, 15.2 \%$ and $10.2 \%$ (see Table 1 ).

Table 1. New bra sizes with high population coverage selected for the study

\begin{tabular}{|c|c|c|c|c|c|c|c|c|}
\hline \multirow{2}{*}{$\begin{array}{l}\text { Undeband size } \\
\text { (cm) }\end{array}$} & \multicolumn{8}{|c|}{ Cup size } \\
\hline & $\mathrm{AA}^{*}$ & $A^{*}$ & $\mathrm{~B}^{\star}$ & $\mathrm{C}^{*}$ & $D^{*}$ & $E^{*}$ & $\mathrm{~F}^{\star}$ & $\mathrm{G}^{*}$ \\
\hline 65 & & & & & & & & \\
\hline 70 & & $6.2 \%$ & $5.0 \%$ & $5.6 \%$ & & & & \\
\hline 75 & & $13.4 \%$ & $15.2 \%$ & $10.2 \%$ & $6.5 \%$ & & & \\
\hline 80 & & $8.1 \%$ & $7.1 \%$ & $7.5 \%$ & $5.0 \%$ & & & \\
\hline 85 & & & $4.3 \%$ & $2.8 \%$ & $3.1 \%$ & & & \\
\hline 90 & & & & & & & & \\
\hline 95 & & & & & & & & \\
\hline 100 & & & & & & & & \\
\hline
\end{tabular}

\subsection{Selection of breast measurements}

In order to identify the key measurements for bra pattern design and to update the new bra sizing, Pearson correlation analysis was conducted on the 103 measurements, which were once used for developing the new bra sizing system with the DWR. 35 measurements are selected as the variables for this study as they have a correlation coefficient more than 0.5 , which means they have a systematic association with the DWR. These measurements included breast circumference, height, width, thickness, angle, surface distance, volume and curvature, which were extracted from the breast cross-section, vertical-section, side view profile, the breast bottom curve (see figure 3). Among them, two measurements, D113RF Angle B of right breast cross-section $\left(-.966^{\star *}\right)$ and C006 Calculated chubbiness ratio of the breast $1\left(.902^{* *}\right)$ have high systematic association with the DWR for their correlation coefficients both more than 0.8 .

To better study the relationship between measurements and bra pattern making, five measurements relevant to the bra pattern design in the practical manufacturing were incorporated into the data set based on the comments from the experienced bra designers, including M046 vertical arc length from right to underbust (BL UBL), D060 Horizontal plane distance between inner-most point of bust, D083 Centre bridge height, D110 width of the breast and D120 height of the breast.
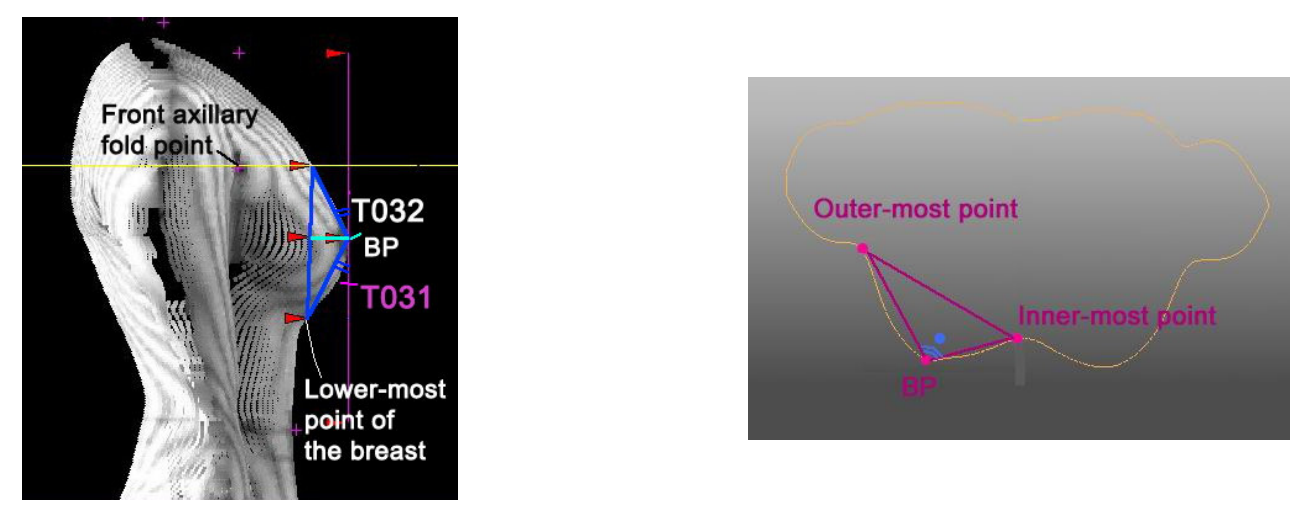

Measurements obtained from the breast vertical-section

Angle $\mathrm{B}$ of right breast cross-section

Fig. 3. 3D variables measured from the vertical-section and cross-section of the bust point 


\subsection{Principal component factor analysis}

To determine the factors and related key measurements to be used in the description of breast shapes during the process of bra cup pattern design, principal components factor analysis depending on varimax rotation was made to identify the groups of measurements from 40 selected variables described in 3.2. The result of the scree plot of initial eigenvalues indicated that five to seven factors might be optimal, and the analysis results revealed that a total of five factors could explain $78.2 \%$ of the variance (see Table 2).

Each factor could be labeled according to the estimated factor loadings and communality results. The first factor was labeled as "overall inner breast shape", factor 2 as "width of breast area", factor 3 as "breast vertical-section profile", factor 4 as "breast cross-section profile", factor 5 as "overall outer breast shape". It can be found that all the five factors, from factor 1 to factor 5 , account for similar percentage of the total variance from $16.64 \%$ to $14.17 \%$. The five factors all play the relatively important role in the bra pattern design.

Table 2. Summary of principal components factor analysis based on five factors

\begin{tabular}{l|ccccc}
\hline & Factor 1 & Factor 2 & Factor 3 & Factor 4 & Factor 5 \\
\hline Loaded measurements & 9 & 10 & 6 & 9 & 6 \\
\hline Initial eigenvalues & 16.66 & 5.55 & 4.03 & 3.03 & 1.98 \\
\hline Rotation sums of squared loadings & 6.65 & 6.62 & 6.49 & 5.82 & 5.67 \\
\hline $\begin{array}{l}\text { \% of variance explained } \\
\begin{array}{l}\text { Cumulative proportion of total sample } \\
\text { variance explained (\%) }\end{array}\end{array}$ & 16.64 & 16.57 & 16.24 & 14.55 & 14.17 \\
\hline
\end{tabular}

According to estimated factor loadings of each measurement in each factor, with such measurements as angle, area, volume and ratio eliminated, the measurements with the estimated factor loading over 0.5 were defined as the key referred measurements for bra pattern making, including D105RF Width of the breast_a_inner, D108RF Inner arc length of right breast (Horizontal plane), D083RF Centre bridge height, M046 Vertical arc length from right bust point to underbust (BP UBL), D059RG Bust point width, D136RF Total arc length of right breast (Slanting plane), D085RF Total arc length of right breast root, D107RF Outer arc length of left breast (Horizontal).

\subsection{Key measurements exploration}

Although individuals distributing into the same new bra size have the same DWR, they will be likely to be different in the width of the breast and the depth of the breast. Moreover, for the individuals in each size, their data distribution of the 40 measurements used in this study showed different deviation from the size centre. Some individuals close to the size centre point and some distant from it.

To further improve the efficiency of the new bra sizing system in practice, the study assessed each individual in the 14 sizes, retaining the individuals close to the centre point of each size, eliminating those individuals on the fringe. The notion of a distance function defined as a dissimilarity measure was used to assess the straight-line distance between individuals and the centre points of their assigned new bra sizes, depending on the five-dimensional factor scores. Squared Euclidean distance was used to assess the factor score aggregate dissimilarity. Suppose that $d\left(x_{n}, y_{s}\right)$ represents the straight-line distance between an individual $n$ and her assigned bra size $s$, which can be calculated by a sum of squared dissimilarities over each of the $f$ factors:

$$
d\left(x_{n i}, y_{s i}\right)=\sum_{i=1}^{f}\left[d_{i}\left(x_{n i}-y_{s i}\right)\right]^{2} \text {, }
$$

where $x_{n i}$ is the factor score of ith factor of nth individual, and $y_{s i}$ is the mean factor score of the th factor of the sth size category assigned to the $n$th individual. The absolute dissimilarity magnitudes were calculated using equation (1) to assess dissimilarity distance value of individuals.

For the data obtained with dissimilarity distance measure for each size, Explore in Descriptive Statistics was used to calculate percentiles of 11 measurements (including the DWR, the width of the breast, the depth of the breast, and eight measurements extracted in 3.3). According to the calculation results, the individuals with the value of the total factor score dissimilarity ranked at the last $10 \%$ were eliminated. For the 11 key measurements, mean values were obtained from the remaining $90 \%$ individuals in each size. 


\section{Discussions}

According to the mean values obtained in 3.4 and the mean analysis results with M-Estimators, the 14 sizes in the new bra sizing system were updated detailedly so as to have a convenient application in the practical designing process. Apart from the two key measurements (underbust girth and the DWR), which were used to establish the new bra sizing system, the width of the breast and the depth of the breast were added into the new sizing system as the control measurements, and eight measurements closely related to the breast shape such as D105RF Width of the breast_a_inner, were added as the referred measurements in the process of bra pattern making.

Table 3 Updated 14 sizes of the new bra sizing

\begin{tabular}{|c|c|c|c|c|c|c|c|c|c|c|c|}
\hline Sizes & $\mathrm{KM}$ & CM 1 & $\mathrm{CM} 2$ & RM 1 & RM 2 & RM 3 & RM 4 & RM 5 & RM 6 & RM 7 & RM 8 \\
\hline $70 A^{*}$ & 0.30 & 13.1 & 4 & 7.0 & 7.2 & 5.5 & 5.9 & 17.6 & 16.2 & 20.2 & 8.7 \\
\hline $70 B^{*}$ & 0.34 & 14.0 & 4.8 & 7.7 & 7.9 & 5.6 & 6.7 & 18.0 & 18.0 & 21.1 & 9.6 \\
\hline $70 C^{*}$ & 0.38 & 14.0 & 5.3 & 8.2 & 8.3 & 5.4 & 6.7 & 18.8 & 17.6 & 21.0 & 9.6 \\
\hline $75 A^{*}$ & 0.30 & 14.1 & 4.3 & 7.4 & 7.6 & 5.4 & 6.2 & 18.5 & 17.4 & 21.2 & 9.1 \\
\hline $75 B^{*}$ & 0.34 & 14.2 & 4.8 & 7.8 & 8.2 & 5.7 & 6.7 & 18.7 & 18.4 & 21.3 & 9.7 \\
\hline $75 C^{*}$ & 0.38 & 14.0 & 5.3 & 8.0 & 8.3 & 5.4 & 7.0 & 19.2 & 18.7 & 21.5 & 10.1 \\
\hline $75 D^{*}$ & 0.42 & 14.2 & 5.9 & 8.2 & 8.7 & 5.2 & 7.6 & 20.0 & 19.0 & 21.8 & 10.5 \\
\hline $80 A^{*}$ & 0.30 & 14.7 & 4.4 & 8.1 & 8.3 & 5.8 & 6.4 & 18.9 & 18.4 & 22.5 & 10.0 \\
\hline $80 \mathrm{~B}^{*}$ & 0.34 & 14.8 & 4.8 & 8.2 & 8.6 & 5.4 & 6.6 & 19.0 & 19.0 & 21.9 & 10.4 \\
\hline $80 C^{*}$ & 0.38 & 14.8 & 5.6 & 8.3 & 8.7 & 5.4 & 7.2 & 19.9 & 19.8 & 22.6 & 10.6 \\
\hline $80 D^{*}$ & 0.42 & 14.6 & 6.1 & 8.4 & 8.9 & 5.2 & 7.4 & 20.2 & 20.0 & 22.0 & 10.7 \\
\hline $85 \mathrm{~B}^{\star}$ & 0.34 & 15.8 & 5.4 & 8.6 & 9.0 & 5.5 & 7.1 & 20.6 & 20.2 & 23.5 & 10.6 \\
\hline $85 C^{*}$ & 0.38 & 16.0 & 6.1 & 8.8 & 9.2 & 5.7 & 7.5 & 21.0 & 21.2 & 24.0 & 12.0 \\
\hline $85 D^{*}$ & 0.42 & 15.7 & 6.6 & 8.6 & 9.1 & 4.9 & 7.8 & 20.9 & 20.4 & 23.5 & 11.4 \\
\hline
\end{tabular}

$\mathrm{KM}$ (Key measurement), the DWR (the depth of the breast/ the width of the breast)

$\mathrm{CM}$ (controlled measurement) 1, the width of the breast

$\mathrm{CM}$ (controlled measurement) 2, the depth of the breast

RM (referred measurement) 1, D105RF Width of the breast_a_inner

RM (referred measurement) 2, D108RF Inner arc length of right breast (Horizontal plane)

RM (referred measurement) 3, D083RF Centre bridge height,

RM (referred measurement) 4, M046 Vertical arc length from right bust point to underbust (BP UBL),

RM (referred measurement) 5, D059RG Bust point width,

RM (referred measurement) 6, D136RF Total arc length of right breast (Slanting plane),

RM (referred measurement) 7, D085RF Total arc length of right breast root,

RM (referred measurement) 8, D107RF Outer arc length of left breast (Horizontal).

It can be noted that some regularity occurred in the data structure in sizes of $75 A^{*}-75 D^{*}$ and $80 A^{*}-80 C^{*}$, which all have the relative high coverage rate among subjects. For example, subjects with the same underbust girth have the similar width of the breast, and the measurements of RM4 (M046 Vertical arc length from right bust point to underbust (BP UBL)) and RM6 (D136RF Total arc length of right breast (Slanting plane)) increase gradually from cup $A^{*}$ to cup $D^{*}$.

\section{Conclusion}

To further improve the application effect of the new bra sizing system in the practical bra design and manufacture, among the most-frequently used 14 sizes in the market, this article identified 40 measurements out of 103 measurements that used for developing the new bra sizing system depending on the correlation coefficient analysis results with the DWR. The five factors describing the breast shape were then derived from the 40 measurements by using principal component factor analysis. They are factor 1 "overall inner breast shape", factor 2 "width of breast area", factor 3 "breast vertical-section profile", factor 4 "breast cross-section profile", and factor 5 "overall outer breast shape". Moreover, eight measurements closely related to the five factors were extracted as referred measurements for the bra pattern making.

Based on the new bra sizing, the study firstly added the width of the breast and the depth of the breast as the control measurements and eight referred measurements as complementarities, and provided effective quantitative data results for each of 14 sizes for further bra pattern making investigation. 


\section{Acknowledgments}

The authors would like to acknowledge the funding support from ITS/028/03 (Development of innovative apparel products and evaluation technology) project of ITF (Innovation and Technology Fund), and from AJ2009-11 of Beijing Municipal Commission of Education. We also like to express our gratitude to AIMER HEC-BICT (Aimer Human Engineering Research Centre of the Beijing Institute of Clothing Technology) for the 3D body scan data.

\section{References}

1. Young, V. L., (1995): "The efficacy of breast augmentation: Breast size increase, patient satisfaction, and psychological effects (Letter) (Reply)", Plastic \& Reconstructive Surgery, Vol.96, pp.1237-1238.

2. Lipton, B., (1996): “Are you wearing the wrong size bra?", Ladies Home Journal, Vol3, pp.46.

3. Boyes, K., (1996): "Buying the perfect bra", Good Housekeeping, Vol.8, pp.50.

4. Greenbaum, A. R., Heslop, T., Morris, J., \& Dunn, K. W., (2003): "An investigation of suitability of bra fit in women referred for reduction mammaplasty", British Journal of Plastic Surgery, Vol.3, No.56, pp.230-236.

5. Armstrong, H. J., (1987): Pattern Marking for Fashion Designer, New York: Harper Collins.

6. Bouman, F. G., (1970): "Volumetric measurement of the human breast and breast tissue before and during mammaplasty", British Journal of Plastic Surgery, Vol.23, pp.263.

7. Campaigne, B. N., Katch, V. L., Freedson, P. et al., (1979): "Measurement of breast volume in females: Description of a reliable method", Annals of Human Biology, Vol.6, pp363-367.

8. Grossman, A. J., \& Roudner, L. A., (1980): "A simple means for accurate breast volume determination", Plastic \& Reconstructive Surgery, Vol.66, pp.851.

9. Ellenbogen, R., (1978): "A new device to assist in sizing breasts", Annals of Plastic Surgery, Vol.3, No.1, pp.333.

10. Martin, R., (1957): Lehrbuch der Anthropologie, Third edition, Fischer: Jena.

11. Wacoal Corp., (1995): Golden canon, Unpublished internal document, Japan.

12. Wacoal Crop., (2001): Curve underwire and its using material, Japan Patent U2001-131807.

13. Bulstrode, N., Bellamy, E. and Shrotria, S., (2001): "Breast volume assessment: comparing five different techniques", The Breast, Vol.10, pp.117-123.

14. Kovacs, L., Eder, M., Hollweck, R., Zimmermann, A., et al., (2007): "Comparison between breast volume measurement using 3D surface imaging and classical techniques", The Breast, Vol.16, pp.137-145.

15. Lee, H. Y., Hong, K., \& Kim, E. A., (2004): "Measurement protocol of women's nude breasts using a 3D scanning technique", Applied Ergonomics, Vol.35, pp.353-359.

16. Lee, H. Y., \& Hong, K., (2007): "Optimal brassiere wire based on the 3D anthropometric measurements of under breast curve", Applied Ergonomics, Vol.3, No.38, pp.377-384.

17. Zheng, R., Yu, W. \& Fan, J., (2008): "A new approach to measure women's breast volume using 3D body scan data", In Proceedings of Applied Human Factors and Ergonomics International Conference 2008, CD format.

18. Zheng, R., Yu, W. \& Fan, J., (2007): "Development of a new Chinese bra sizing system based on breast anthropometric measurements", International Journal of Industrial Ergonomics, Vol.37, pp. 697-705.

19. Pechter, E. A., (1998): "A new method for determining bra size and predicting postaugmentation breast size", Plastic \& Reconstructive Surgery, Vol.4, No.102, pp.1259-1265.

20. Zheng, R., (2007): "Breast sizing and development of 3D seamless bra", Unpublished PhD thesis, Hong Kong Polytechnic University, Hong Kong. 